\title{
Design \& Development of Thermoelectric Cooler Using Peltier Plate
}

\author{
Narendra patil ${ }^{1}$, Munot Hrushabh ${ }^{2}$, Chetan Patil ${ }^{3}$, Omkar Patil ${ }^{4}$, Tushar Mahale ${ }^{5}$, \\ Deepesh Kumbhakarna ${ }^{6}$ \\ Student, Department of Mechanical Engineering, Trinity Academy of Engineering, Pune 1,2,3,4,5 \\ Assistant Professor, Department of Mechanical Engineering, Trinity Academy of Engineering, Pune ${ }^{6}$
}

\begin{abstract}
The increase in demand for refrigeration globally in the field of air-conditioning, food preservation, medical services, vaccine storages, and for electronic components temperature control led to the production of more electricity and consequently an increase in the $\mathrm{CO} 2$ concentration in the atmosphere which in turn leads to global warming and many climatic changes. Thermoelectric refrigeration is a new different because it can reduce the use of electricity to produce cooling effect and also meet today's energy challenges. Therefore, the need for thermoelectric refrigeration in developing countries is very high where long life and low maintenance are needed. The objectives of this study is to develop a working thermoelectric refrigerator to cool that utilizes the Peltier effect to cool and maintain a selected temperature range of $50 \mathrm{C}$ to $250 \mathrm{C}$. The design requirements are to cool this volume to temperature within a short time and provide retention of at least next half an hour. The design and development of thermoelectric refrigerator for required applications are presented.
\end{abstract}

Keywords: Thermoelectric Module, Peltier plate, Heat sink, Blower

\section{INTRODUCTION}

The conventional cooling systems are used now a days are requires the refrigerant whose phase change takes place in heat exchanging and compressor are required for the compression of the refrigerant. The compressor required more power and space. The refrigerant is also not eco-friendly and increases the global warming and the major cause of ozone layer depletion. The mini Eco-friendly refrigerator is based on the PELTIER EFFECT and a thermoelectric device called Peltier device is used for the cooling purpose. In the MEF-Refrigerator there is no need of compressor and refrigerant. Semiconductor thermoelectric coolers (also known as Peltier coolers) off temperature control (\&lt; \pm $0.1^{\circ} \mathrm{C}$ ) can be achieved with Peltier coolers. However, the efficiency of the conventional refrigerators is medium. Thus, they are used in medium-scale applications where their unique advantages outweigh their low efficiency. Although some wide-ranging applications have been considered (on submarines and surface vessels), Peltier coolers are generally used in applications where small \& medium size is needed and the cooling demands are not too great but it have some good demand in market, such as for cooling electronic components.

\section{LITERATURE SURVEY}

Conventional cooling systems such as those used in refrigerators utilize a compressor and a working fluid to transfer heat. Thermal energy is absorbed and released as the working Fluid undergoes expansion and compression and changes phase from liquid to vapor and back, respectively. Semiconductor thermoelectric coolers (also known as peltier coolers) offer several advantages over conventional systems.

Title: Jonathan Michael Schoenfeld et at [1] in his thesis submitted on integration of a thermoelectric sub cooler in 2008.

In this paper There are two general research areas focused on increasing TEC performance; materials Researchon thermoelectric semiconductors and system level assembly and heat dissipation techniques. The former is focused on developing advanced thermoelectric materials with superior thermoelectric properties. The most important parameter of a thermoelectric semiconductor is the figure of merit, Z, which is given by $\alpha 2 /(\mathrm{k} \rho \mathrm{e})$. Each of these properties is temperature dependent so often the of merit will be given at a particular temperature in the dimensionless form, ZT. Increasing the figure of merit directly results in an increase in the optimum COP of a TEC. The most common thermoelectric semiconductor in todays TECs is Bismuth Telluride (Bi 2Te 3), which has a ZT of $\sim 0.9$ at $300 \mathrm{~K}$. 


\section{International Advanced Research Journal in Science, Engineering and Technology}

Vol. 6, Issue 3, March 2019

Title: Bass et al. (2004) [2] investigated the use of multi-layer quantum well (MLQW) thermo electrics in a cooling application.

In this paper MLQW thermoelectric material is a composite of thin layers of alternating semiconductor material with differing electronic band gaps deposited on a substrate. In this way, the thermal and electrical conductivity of the material can be decoupled. The non-dimensional figure of merit of such composite materials has been determined experimentally to be as high as 3 or 4 . Theoretical analysis predicted COPs as high as 5 at a $\Delta$ Tm of $20 \mathrm{~K}$. A TEC utilizing MLQW thermoelectric material is still under development. It can be expected that the additional manufacturing costs of such a module would be substantial.

Title ; Chain and Chen et al.(2005) [3] investigated the use of a micro channel heat sink on a TE module used to cool a water tank.

In this paper The micro channels were etched into a silicon wafer with a glass cover plate. Four micro channel heat exchangers were fabricated with a differing number of ports and hydraulic diameters $(\mathrm{D} \mathrm{h})$, from 89 ports at a $\mathrm{D}$ h of 65 $\mu \mathrm{m}$ to 44 ports at a $\mathrm{D} \mathrm{h}$ of $150 \mu \mathrm{m}$. Water was pumped at flow rates ranging from $289-10,702 \mathrm{ml} / \mathrm{h}$ to remove the heat from the hot side of the module. The micro channel was placed on top of a $4 \mathrm{~cm} \mathrm{x} 4 \mathrm{~cm}$ TE module. The lowest measured thermal resistance for the heat sink was $1.68 \mathrm{~K} / \mathrm{W}$. The authors suggested that the thermal resistance could be reduced to $0.5 \mathrm{~K} / \mathrm{W}$ by increasing 6 the aspect ratio of the micro channel ports and by using a more conductive material like copper.

\section{OBJECTIVES}

The material used to making the model is lower graded and economical because the cost of product will less as possible as, which will play an advantage should this product be large numbers into the market.

The discussion of this project will include the positive aspects of the project and its flaws, and will explain how the latter can be overcome should this project continue in the future.

-To provide cooling system in rural area.

-To reduce the overall cost of refrigeration to cool water.

-To investigate methods to increase the efficiency in order to conserve power.

\section{COMPONENTS}

These are the following components of thermoelectric cooler :

1. Peltier module

2. Heat sink

3. Blower

\section{Thermoelectric Module :}

A thermoelectric module thus uses a pair of fixed junctions into which electrical energy is Applying causes one junction to become cold while the other becomes hot. Because thermoelectric cooling is a form of good refrigeration, it has the advantage of being compact and long lasting. It uses no moving parts except for some fans, employs no fluids, and do not require bulky piping and mechanical compressors used in vapour-cycle cooling systems. Such sturdiness favour thermoelectric cooling over conventional refrigeration in certain situations. The compact size and weight requirements, as well as portability in the design, rule out the use of conventional refrigeration.

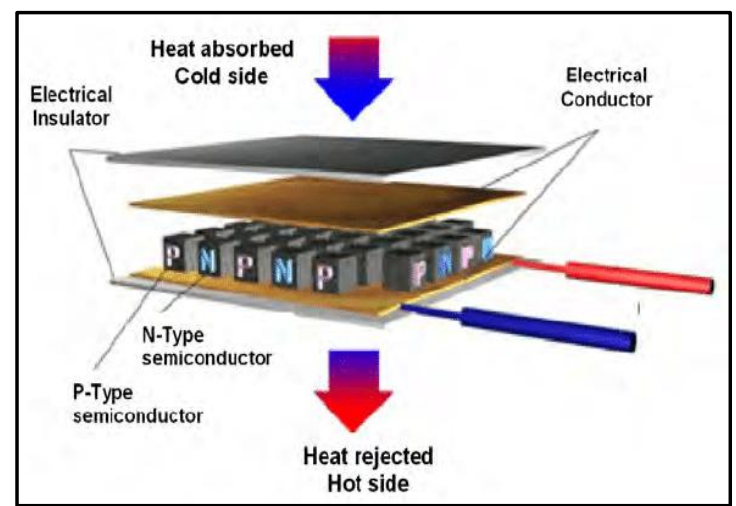

Fig 1. Thermoelectric module 


\section{International Advanced Research Journal in Science, Engineering and Technology}

Vol. 6, Issue 3, March 2019

\section{WORKING}

In the figure shows working principle of thermoelectric module shows a simple diagram of peltier. The elements of semiconductor, primarily Bismuth Telluride, heavily doped to create either an excess (n-type) or deficiency (p-type) of electrons. If the peltier module gets cooled on one side it transfers its cooling to the heat sink used on that same side.

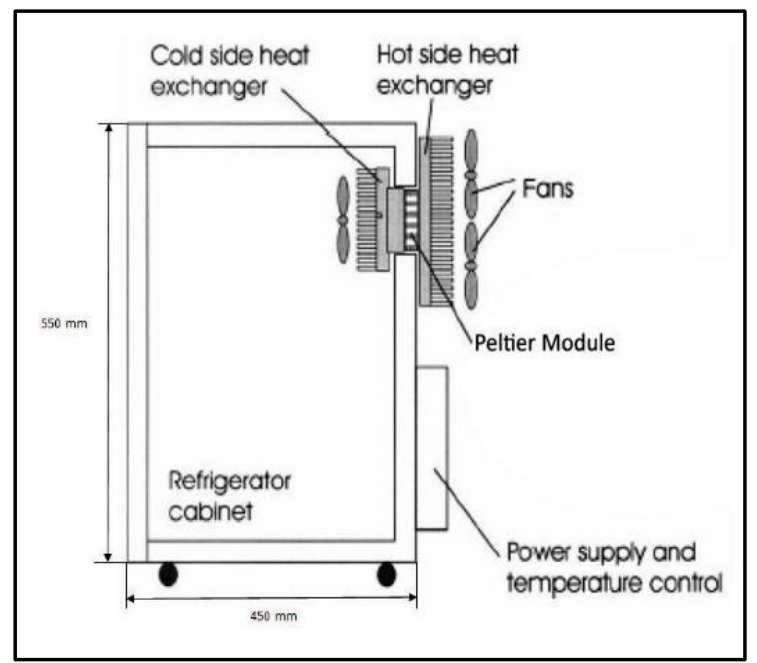

Fig 2.Working Diagram

Due to this transfer of cooling, the fans provided to the heat sinks spred the cool air and gives out to the required place needed for cooling. The other side fan on opposite side of the peltier module draws the hot air from the heat sink and expels out to the atmosphere in order to decrease the temperature of the peltier module. In this way the whole system works.

\section{ADVANTAGES}

a. Compact size : Very little space is required by the cooling system. The thermoelectric module is the size of a matchbook.

b. Lightweight : 36 qt. capacity unit weighs only 17 lbs. PORTABLE: Carries with one hand and is unaffected by motion

c. Lower price : $20 \%$ to $40 \%$ less expensive than compressor or absorption units.

\section{REFERRENCES}

[1]. Jonathan Michael Schoenfeld, Master of Science,2008 Computational Model for Refrigerators Based on Peltier Effect Application, Applied Thermal Engineering, Vol. 25, No. 13, pp. 3149-3162.

[2]. Bass et al. (2004), Multi-layer quantum well (MLQW) thermo electrics in a cooling application International journal of research in aeronautical and mechanical engineering ISSN (online): 2321-3051

[3]. Chain and Chen Vol. 120 (2011) Performance Prediction and Irreversibility Analysis of a Thermoelectric Refrigerator with Finned Heat Exchanger Wuhan 430033, P.R. China, ACTA PHYSICA POLONICA No.03

[4]. Riff at and Qiu (2005) Air conditioning systems with an air and water cooled heat sink International Journal of Emerging Technology and Advanced Engineering Volume 3, Special Issue 3: ICERTSD 2013, Feb 2013, ISSN 2250-245

[5]. Zhang H Y (2010), A General Approach in Evaluating and Optimizing Thermoelectric Coolers, Int. Journal of Refrigeration, Vol. 33, No. 10, pp. 1187-1196.

[6]. Gaurav Maradwar, Fabrication And Analysis Of Problems In Thermoelectric Refrigerator International Journal Of Core Engineering \& Management(IJCEM) Volume 1, Issue 2, May 2014, ISSN:2348 9510 